\title{
High-flow nasal cannula for bronchiolitis in an ICU and step-down unit
}

\author{
Madeleine M. Genereux MD¹, Morgan J. Sims MD², Bethany J. Wolf PhD ${ }^{3}$, Ian D. Kane MD²
}

M.M. Genereux, M.J. Sims, B.J. Wolf, I.D. Kane. High-flow nasal cannula for bronchiolitis in an ICU and step-down unit. Can J Respir Ther 2020;56:5-6 doi: 10.29390/cjrt-2019-023.

Children with bronchiolitis often require hospital admission for supportive care including standard nasal cannula oxygen. At many hospitals, infants who require high-flow nasal cannula (HFNC) respiratory support must be admitted to an intensive care unit (ICU). More recently, however, studies have shown that the use of HFNC outside of the ICU setting is effective and well tolerated [1-4]. At our institution, infants with bronchiolitis who require HFNC may be admitted to a lower acute step-down ICU (SDU) at the attending physician's discretion. Historically, admission to the SDU has been used for infants felt to be at low risk for adverse events such as progression to continuous positive airway pressure (CPAP) support, bi-level positive airway pressure (BiPAP) support, or intubation. The evaluation of infants with bronchiolitis is difficult because of the subjective nature of assessing respiratory distress, and a recent study identified risk factors that predict the need for escalation of care beyond nasal cannula [5]. However, a small retrospective review that sought to predict which patients on HFNC would require increased respiratory support and intubation showed that easily identifiable markers such as patient age and history of prematurity were not predictive of failure among bronchiolitic patients being treated with HFNC [6].

We used a retrospective study design to evaluate the clinical characteristics and adverse events of infants on HFNC for bronchiolitis who were admitted to our standard ICU or our SDU. By assessing for differences in clinical characteristics and outcomes among patients admitted on HFNC to our ICU and SDU, we also sought to determine if clinicians could select a cohort of infants on HFNC who were at lower risk for adverse events. We included patients aged 1 month through 2 years admitted with bronchiolitis on HFNC and excluded those with significant prematurity or congenital heart or lung disease. Incidence of adverse outcomes was defined as a medical emergency team response, or escalation of respiratory support to CPAP, BiPAP, or intubation.

Sixty-one infants met our inclusion criteria and were admitted from the emergency department to the ICU (26 patients) or to the SDU (35 patients) on HFNC. Clinical characteristics and adverse events for each group are shown in Table 1 . The mean patient age was significantly lower in the ICU group compared with the SDU group (median 3.5 vs. 9.8 months, $p=0.045$ ) and children admitted to the ICU had a higher initial flow of HFNC compared with those admitted to the SDU (median $8 \mathrm{~L} / \mathrm{min}$ vs. $6 \mathrm{~L} / \mathrm{min}$ ). The rate of adverse events was not different between those admitted to the ICU and those admitted to the SDU. Progression to CPAP or intubation occurred in only 2 patients in each group. Median hospital length of stay was 4 days for the ICU group and 3 days for the SDU group which was not statistically significant.

There is growing literature to suggest HFNC is safe for use outside of an ICU setting. However, concerns remain that children who require HFNC may need escalation of care that prompts admission to an ICU. If clinicians could identify a lower risk cohort of infants with

\section{TABLE 1}

Admission on HFNC to the ICU versus the SDU

\begin{tabular}{lccc}
\hline & $\begin{array}{c}\text { ICU } \\
(\boldsymbol{n}=\mathbf{2 6})\end{array}$ & $\begin{array}{c}\text { Step down } \\
(\boldsymbol{n}=\mathbf{3 5})\end{array}$ & $\boldsymbol{p}$ \\
\hline Demographics & & & \\
Race, $n$ (\%) & & & 0.673 \\
$\quad$ White & $9(34.6)$ & $14(40.0)$ & \\
$\quad$ African American & $11(42.3)$ & $16(45.7)$ & \\
$\quad$ Other & $6(23.1)$ & $5(14.3)$ & \\
Sex, male, $n$ (\%) & $17(65.4)$ & $19(54.3)$ & 0.383 \\
Age, month, median (IQR) & $3.5(2.0-9.9)$ & $9.8(2.4-16.0)$ & 0.045 \\
Admission clinical characteristics & & & \\
Documented tachypneic, yes, $n(\%)$ & $20(76.9)$ & $29(82.9)$ & 0.564 \\
Documented retractions, yes, $n(\%)$ & $24(92.3)$ & $33(94.3)$ & 1.000 \\
IV fluids started in ED, yes, $n(\%)$ & $24(92.3)$ & $32(91.4)$ & 1.000 \\
CXR in ED, yes, $n$ (\%) & $23(88.5)$ & $25(71.4)$ & 0.128 \\
Viral process on CXR in ED, & $11(47.8)$ & $18(72.0)$ & 0.087 \\
yes, $n$ (\%) & & & \\
$\quad$ Infiltrate on CXR in ED, yes, $n(\%)$ & $10(43.5)$ & $7(28.0)$ & 0.367 \\
Positive for RSV, yes, $n$ (\%) & $16(61.5)$ & $18(51.4)$ & 0.432 \\
Received albuterol in ED, yes, $n(\%)$ & $14(53.9)$ & $20(57.1)$ & 0.798 \\
Day of illness, median (IQR) & $3(2-6)$ & $3(2-4)$ & 0.231 \\
Litre of HFNC, median (IQR) & $8(6-8)$ & $6(4-7)$ & $<0.001$ \\
FiO ${ }_{2}$ of HFNC, median (IQR) & $45(35-90)$ & $40(30-50)$ & 0.149 \\
Adverse outcomes & & & \\
MET, yes, $n$ (\%) & $0(0.00)$ & $2(5.71)$ & 0.503 \\
Progression to CPAP or intubation, & $2(7.69)$ & $2(5.71)$ & 1.000 \\
yes, $n$ (\%) & & & \\
Hospital LOS, median (IQR) & $4(3-5)$ & $3(3-6)$ & 0.755 \\
Length of stay >5 days, $n$ (\%) & $4(15.4)$ & $9(25.7)$ & 0.33 \\
\hline
\end{tabular}

Note: HFNC, high-flow nasal canula; ICU, intensive care unit; SDU, step-down ICU; ED, emergency department; CXR, chest X-ray; RSV, Respiratory Syncytial Virus; MET, medical emergency team rapid response; LOS, length of stay.

${ }^{1}$ Department of Pediatrics, Medical University of South Carolina, Charleston, SC, USA

${ }^{2}$ Department of Pediatrics, Division of Pediatric Emergency Medicine, Medical University of South Carolina, Charleston, SC, USA

${ }^{3}$ Department of Public Health Services, Medical University of South Carolina, Charleston, SC, USA

Correspondence: Madeleine Genereux, Medical University of South Carolina, Pediatric Emergency Medicine, 135 Rutledge Avenue, MSC 561, Charleston, SC 29425, USA; Tel: 843-754-9037.E-mail: genereux@musc.edu

Published online at https://www.cjrt.ca on 28 February 2020

OPEN ACCESS

This open-access article is distributed under the terms of the Creative Commons Attribution Non-Commercial License (CC BY-NC) (http:// creativecommons.org/licenses/by-nc/4.0/), which permits reuse, distribution and reproduction of the article, provided that the original work is properly cited and the reuse is restricted to noncommercial purposes. For commercial reuse, contact editor@csrt.com 
bronchiolitis who required HFNC, this could support the use of HFNC in selected cases outside of an ICU setting. In our study the incidence of adverse outcomes was low and was not clinically significant between patients admitted on HFNC to the ICU and those admitted on HFNC to the SDU. Our results, particularly our low rate of adverse events, were influenced because we excluded children with prematurity and pre-existing chronic lung or congenital heart disease. Our study had limited power to detect differences in adverse outcomes owing to its sample size. A larger study without exclusion criteria and increased frequency of adverse outcomes may be better able to assess whether clinician impression can identify low-risk children admitted on HFNC for bronchiolitis. Our results support the assertion that the evaluation of bronchiolitis remains subjective; despite this, our overall complication rate was low.

\section{FUNDING SOURCE}

This work was funded in part by support funding from the South Carolina Clinical \& Translation Research Institute, Medical University of South Carolina's CTSA, NIH/NCCR Grant Number UL1TR001450.

\section{REFERENCES}

1. Milani GP, Plebani AM, Arturi E, et al., Using a high-flow nasal cannula provided superior results to low-flow oxygen delivery in moderate to severe bronchiolitis. Acta Paediatr 2016;105: e368-72. doi: 10.1111/apa.13444.

2. Wing R, James C, Maranda LS, et al. Use of high-flow nasal cannula support in the emergency department reduces the need for intubation in pediatric acute respiratory insufficiency. Pediatr Emerg Care 2012;28: 1117-23. doi: 10.1097/PEC.0b013e31827122a9.

3. Kallappa C, Hufton M, Millen G, et al. Use of high flow nasal cannula oxygen (HFNCO) in infants with bronchiolitis on a paediatric ward: A 3-year experience. Arch Dis Child 2014;99: 790-1. doi: 10.1136/ archdischild-2014-306637.

4. Bressan S, Balzani M, Krauss B, et al. High-flow nasal cannula oxygen for bronchiolitis in a pediatric ward: A pilot study. Eur J Pediatr 2013;172: 1649-56. doi: 10.1007/s00431-013-2094-4.

5. Franklin D, Babi FE, Schlapbach LJ, et al. A randomized trial of highflow oxygen therapy in infants with bronchiolitis. N Engl J Med 2018;378: 1121-31. doi: 10.1056/NEJMoa1714855.

6. Abboud PA, Roth PJ, Skiles CL, et al. Predictors of failure in infants with viral bronchiolitis treated with high-flow, high-humidity nasal cannula therapy. Pediatr Crit Care Med 2012;13: e343-9. doi: 10.1097/ PCC.0b013e31825b546f. 